\title{
SIDA : faits et chiffres
}

\section{Les nouvelles de ce numéro ont été préparées par: Guillaume Bobrie Jean-Claude Dreyfus Serge Erlinger Axel Kahn}

L'épidémie de SIDA continuant à se développer dans le monde, la revue Science vient de consacrer sa couverture, et plus de la moitié de son sommaire, à faire le point sur les différents aspects de la progression de cette épidémie à la fin de l'année 1987 (Science 1988, $n^{\circ} 4840$, vol.239).

75000 cas avaient été, fin 1987, rapportés dans le monde, dont $70 \%$ aux ÉtatsUnis [1, 3]. Compte tenu du manque d'informations précises sur le nombre réel de malades dans certains pays africains, c'est probablement à 150000 que l'on peut estimer le chiffre réel de cas [1]. Du fait de la durée de l'incubation de la maladie et du nombre actuel probable de sujets infectés non malades dans le monde (non connu avec précision, mais probablement de l'ordre de plusieurs millions, dont un à deux millions rien qu'aux États-Unis), le nombre de malades devrait continuer à croître de manière exponentielle ces quatre prochaines années, atteignant 270000 cas cumulés en 1991 aux ÉtatsUnis, et ce quelle que soit par ailleurs l'évolution de l'épidémie, qui est actuellement, en fait, mal connue. Aux États-Unis, les fréquences de séropositivité chez les candidats au recrutement par l'armée tend à se stabiliser [3], mais, en revanche, l'épidémie flambe en Afrique, principalement dans les grandes villes [1]. Dans certains groupes de prostituées féminines de villes du centre et de l'est africain, la prévalence des séropositivités dépasse $80 \%$. Les modes de transmission continuent d'être extrêmement différents en Europe de l'Ouest et Amérique d'une part, en Afrique de l'autre. Dans le premier groupe, ce sont toujours les homosexuels masculins, les drogués, les hémophiles et leurs partenaires sexuels qui constituent l'énorme majorité des observations. Dans le groupe africain, la transmission est majoritairement hétérosexuelle. La maladie reste rare et sporadique en Europe de l'Est, en Océanie et en Asie.

Les risques d'infection sont directement en rapport avec le nombre de partenaires sexuels. D'autres facteurs de risques à confirmer seraient la prise de contraceptifs chez les femmes, et la non-circoncision chez les hommes. L'existence d'ulcérations génitales et d'infections génitales à Chlamydiae est associée à une plus grande prévalence de la maladie ainsi qu'à une plus grande infectiosité. De même, l'infectiosité augmenterait avec l'évolution de la maladie, les sujets sains séropositifs étant moins infectieux que les malades chez lesquels la prolifération virale est plus active. Cette dernière donnée pourrait être assez inquiétante puisque, augmentant la contagiosité des séropositifs sains actuels, elle pourrait entraîner secondairement une stimulation de l'épidémie [1].

En fonction de tous ces facteurs, le risque individuel de séropositivation en cas de contact sexuel avec un sujet infecté varie selon les cas; il est, sur plusieurs années, de 7 à $23 \%$ chez les conjoints d'hémophiles séropositifs et pourrait atteindre, en Afrique, $10 \%$ pour un contact sexuel unique avec une prostituée cumulant les éléments d'infectiosité (infection et ulcération génitales principalement).

Le risque général d'infection après un contact unique avec un sujet infecté est estimé à $1 \%$ [2]. La contamination de la mère séropositive à l'enfant, principalement durant la période intra-utérine, est importante, de 30 à $65 \%$ des cas.

Enfin, la grande fréquence d'apparition du SIDA chez les sujets infectés par HIV-l se confirme: elle est, de façon cumulative, de $5 \%$ après 
trois ans de séropositivité, $10 \%$ après quatre ans, $15 \%$ après cinq ans, $24 \%$ après six ans et $36 \%$ après sept ans [3].

L'infection par HIV-2 est surtout fréquente en Afrique de l'Ouest et s'étend rapidement. Il est sûr maintenant que HIV -2 est pathogène, mais la fréquence d'apparition des signes cliniques chez les séropositifs reste inconnue.

Les principales mesures prophylactiques susceptibles de diminuer les risques d'infection sont l'utilisation constante de préservatifs masculins (qui ne donne cependant une protection que de $90 \%$ par contact sexuel et environ 62 à $70 \%$ pour une probabilité cumulée d'être infecté après 1000 contacts sexuels) et la réduction du nombre de partenaires sexuels. Encore faut-il, pour qu'elle soit « efficace», que cette réduction soit importante, puisque le risque diminue peu pour un individu passant de 50 à cinq partenaires sexuels sur quelques années, le «bénéfice » devenant important pour une réduction à un partenaire exclusif [2].
A. K.
1. Piot P, Plummer FA, Mhalu FS, Lamboray JL, Chin J, Mann JM. AIDS : an international perspective. Science $1988 ; 239$ : $573-9$.

2. Fineberg HV. Education to prevent AIDS : prospects, obstacles. Science $1988 ; 239: 592-6$.

3. Curran JW, Jaffe HW, Hardy AM, Morgan WM, Selik RM, Dondero TJ. Epidemiology of HIV infection and AIDS in the United States. Science 1988; 239: 610-6.

\section{Pourquoi l'intoxication par la réglisse entraîne-t-elle une hypertension artérielle?}

L'activité gluco- et minéralocorticoïde des stéroïdes est liée à l'existence d'un groupe hydroxyle en Cll. Le cortisol actif est transformé habituellement en cortisone inactive grâce à un complexe enzymatique microsomial, principalement hépatique et rénal, la $11 \beta$ hydroxystéroïde déshydrogénase ( $11 \beta \mathrm{OH}$ $\mathrm{SD})$. Le déficit en cette enzyme aboutit à des concentrations intratissulaires importantes de cortisol, qui agit alors comme un puissant minéralocorticoïde, et à une augmentation d'excrétion urinaire des métabolites du cortisol (aux dépens de celle des métabolites de la cortisone), alors que la cortisolémie reste normale.

Le syndrome d'excès apparent de minéralocorticoïdes est un syndrome rare, responsable chez l'enfant d'une hypertension artérielle avec hypokaliémie et inhibition du système rénine-angiotensine-aldostérone. Il est secondaire à un déficit congénital en $11 \beta \mathrm{OH}-\mathrm{SD}$. Cela augmente le rapport entre les métabolites urinaires du cortisol et ceux de la cortisone. Ce syndrome est corrigé par la dexaméthasone qui supprime la production de cortisol.

L'intoxication par la réglisse entraîne une hypertension artérielle, une hypokaliémie et une inhibition du système rénine-aldostérone. Depuis longtemps, ces anomalies ont été attribuées à l'effet minéralocorticoïde faible de l'acide glycyrrhizinique (provenant de la réglisse). Récemment Stewart et al. [1] ont remarqué l'analogie entre l'intoxication par la réglisse et le syndrome d'excès apparent de minéralocorticoïdes : les manifestations cliniques sont superposables ; dans les deux cas, les antagonistes de l'aldostérone sont efficaces ; dans l'intoxication par la réglisse, la dexaméthasone corrige également les anomalies et celles-ci ne se développent pas chez le sujet insuffisant surrénalien. Ces auteurs ont donc étudié les effets de la prise d'acide glycyrrhizinique pendant dix jours chez sept hommes volontaires sains. Outre les manifestations attendues, ils ont observé une augmentation du cortisol libre urinaire, sans modification du cortisol plasmatique, et une augmentation du rapport métabolites urinaires du cortisol/métabolites urinaires de la cortisone. Ces modifications surviennent pour des concentrations plasmatiques d'acide glycyrrhizinique inférieures à celles nécessaires pour déplacer l'aldostérone de son récepteur - c'est-à-dire pour exercer une activité minéralocorticoïde.

Les conséquences cliniques de l'intoxication par la réglisse pourraient donc bien relever de l'inhibition de la $11 \beta \mathrm{OH}-\mathrm{SD}$, à l'image de ce qu'on observe dans le déficit congénital de cette enzyme.

G. B.

\section{BRÈVES}

L'ichthyose congénitale liée à l'X semble presque constamment secondaire à une délétion du gène de la stéroïde-sulfatase. Cette maladie est due à l'absence de l'enzyme qui dégrade normalement le cholestérolsulfate dont l'abondance dans la peau inhibe la desquamation de la couche cornée, conduisant ainsi à son épaississement caractéristique de l'ich thyose. Dans 40 des 45 familles étudiées jusqu'à présent par différents auteurs [1], la base moléculaire de la maladie est une délétion du gène L'ichthyose congénitale semble à l'heure actuelle l'une des affections dans laquelle la plus haute proportion de mécanismes délétionnels a été observée. Cette particularité s'ajoute aux autres «bizarreries » du gène de la stéroïde-sulfatase : situé à l'extrémité du bras court, il est très proche de la région à transmission pseudoautosomique chez l'homme... et dans cette région chez la souris $(\mathrm{m} / \mathrm{ssuppl}$. au $n^{\circ} 7$, vol. 3, p.11). De plus, il échappe partiellement à l'inactivation au hasard d'un chromosome $\mathrm{X}$ au cours de l'embryogenèse.

[1. Bonifas JM, et al. Proc Natl Acad Sci USA 1987 ; 84 : 9248-51]

1. Stewart PM, Valentino $R$, Wallace AM, Burt D, Shackleton CHL, Edwards CRW. Mineralocorticoid activity of liquorice: 11. beta-hydroxysteroid dehydrogenase deficiency comes of age. Lancet $1987 ; 2: 821-3$. 